\title{
Cellular Memory
}

\section{Contents}

3.1 Maintaining Cellular Fates - 50

3.2 PcG/TrxG System Maintaining Cellular Memory - 51

3.3 Biochemical Characterization and Molecular Function of PcG/TrxG Proteins - 53

3.4 Targeting and Propagation of PcG/TrxG-Controlled Chromatin Domains - 55

3.5 Switching Memory and the Role of Non-coding RNAs - 59

3.6 Losing Memory - 61

References -65 


\section{What You Will Learn in This Chapter}

The identity of cells in an organism is largely defined by their specific transcriptional profile. During cell division, these profiles need to be faithfully inherited to the daughter cells to ensure the maintenance of cell structure and function in a cell lineage. Here, you will learn how two groups of chromatin regulators, the Polycomb group (PcG) and the Trithorax group (TrxG), act in an antagonistic manner to maintain differential gene expression states. Members of the PcG cooperate in large multiprotein complexes to modify histones with repressive marks, resulting in condensed chromatin domains. Conversely, the $\operatorname{TrxG}$ proteins counteract the repressed domains by opening nucleosomal structures and establishing activating histone modifications. PcG and TrxG proteins are evolutionary highly conserved and control diverse processes, such as the identity of stem cells in mammalian development to the process of vernalization in plants.

\subsection{Maintaining Cellular Fates}

Metazoan organisms reach their size and body complexity through a sequential series of cellular specifications intermingled with phases of growth. During early embryonic stages, the body pattern is defined, the germ layers established, and the primordia for the organs delineated. Subsequently, during the process of differentiation the developmental fates are implemented to produce morphological features, organ structures, and functions fulfilling the needs of survival and reproduction of the specific organism. During all these development processes, cells also undergo a large number of divisions, with the requirement to retain the once established developmental fates ${ }^{1}$. To a large part, the fates and identities of cells are written in their differential patterns of gene expression. Hence, after each cell division, these unique transcriptional networks have to be reestablished to maintain cellular identities. This is not a trivial task given the extensive molecular and cellular rearrangements observed during the processes of genome replication and cellular division, where most regulatory proteins and chromatin factors are stripped from the DNA. However, complex mechanisms evolved to retain cellular identities in the wake of these processes, which ensure that developmental decisions are faithfully maintained. In other words, cells seem to have a "cellular memory".

How can the "memory" of cells be revealed? Transplantation experiments have demonstrated that cells and tissues react differently when moved to a foreign neighborhood, depending on their programmed state. Before being determined for a particular identity, transplanted cells often adapt to the identity of their new foreign neighbors. Conversely, when transplanted after having been determined, they retain their original identity even when exposed to a new developmental environment. This shows that there is a specific period in the development of an organism in which cellular programs become fixed and irreversible (Wolpert et al. 2015).

1 Developmental fates are built through consecutive developmental restrictions. Among the first are the allocations to the germ layers (ecdoderm, endoderm and mesoderm). Subsequently, cells receive information to be part of a specific organ or tissue and fulfill a particular structural or functional role. Every step of these decisions have to be memorized and faithfully maintained. 
In Drosophila melanogaster, at metamorphosis, larval cell are eliminated and adult precursor cells become differentiated during the subsequent pupal stage, giving rise to the organs and appendages of the adult fly. This process is triggered by the hormone 20-hydroxyecdysone (ecdysone). The burst of hormone induces a wellorchestrated cascade of transcriptional and post-transcriptional events leading to the differentiation of adult precursor (imaginal) cells. An example being the transformation of the morphologically inert imaginal disc cells which give rise to the highly specialized external cuticular structures, like wings, legs, or eyes. Concomitantly, larval cells and tissues which do not form part of the adult fly become marked for death and are histolyzed during metamorphosis. A classical experiment by Hadorn and collaborators demonstrated that imaginal discs maintain and remember their determined program (Hadorn 1968). Clusters of cells representing the primordia of imaginal discs are defined at early stages of embryogenesis. During subsequent embryonic and larval stages, theses clusters grow in size but do not differentiate. Only at metamorphosis, the cells implement their early defined transcriptional programs and differentiate into the structures and appendages that give rise to the external cuticle of the fly. Fragments of imaginal discs can be transplanted into the fluid of the abdomen of adult flies (hemolymph), which acts like an incubator, allowing the discs to grow in size but not to differentiate. This process can be repeated over many weeks and months, forcing the imaginal disc cells to undergo more cell divisions than they would have done during normal development of a fly. To observe whether the cells also retained their identity under these circumstances, they are transplanted back into a larva prior to metamorphosis. After undergoing metamorphosis, the hatched adult fly contains, in its hemolymph, differentiated cuticular tissue of the same identity as the original imaginal disc. This clearly demonstrates that cells can retain a memory of their programmed state even when placed in a foreign environment and undergoing many cell divisions.

Interestingly, Hadorn and collaborators observed rare cases where the transplanted imaginal disc fragments, after differentiation, produced cuticular structures other than those originally determined. Hence, cells can also "forget" and change fate. They termed this phenomenon "transdetermination". These changes of fate often mimicked phenotypes which had been observed in homeotic mutants. These are mutations in master regulatory genes required to establish the appropriate segmental identities in the fly (Lewis 1978). As such, it was argued that the maintenance of cellular memory is inherently connected to the faithful conservation of the specific expression patterns of master developmental regulators.

\subsection{PcG/TrxG System Maintaining Cellular Memory}

Parallel to the studies of Hadorn, geneticists identified fly mutations displaying multiple homeotic phenotypes. Among these, Ed Lewis and his wife Pam characterized a mutation they named "Polycomb", which they demonstrated to control the homeotic master regulators in the Bithorax Complex (BX-C) (Lewis 1978). In this mutant, the cuticular structures of the second and third leg were transformed towards the identity of the first leg. Given that normally, in males, only the first leg pair depicts socalled sex combs, the fact that in the mutant all legs developed this morphological 
marker pointed to a deregulation of the underlying segmental identity imposed by the homeotic master regulators. Over time, a number of other genes with a similar phenotype were isolated and classified as "Polycomb group (PcG)" genes. Their phenotype indicated that they all act as repressors of homeotic master regulators. Interestingly, early patterns of homeotic gene expression were not disturbed but they were only at later stages (Struhl and Akam 1985). This suggested that the products of the PcG were not responsible for establishing the segment-specific expression patterns of master regulators, but for maintaining them appropriately expressed for the rest of development. Additionally, mutations in PcG genes also increased the rate of transdetermination events observed in transplantation experiments, suggesting that this group is part of the system maintaining cellular identities (Grossniklaus and Paro 2014).

In Drosophila, mutations like Trithorax, which mimic loss-of-function homeotic mutations, were identified. Further genetic analyses also revealed the existence of an entire group of genes acting as repressors of the additional sex combs phenotype found in PcG mutants. Mutations in these genes reversed or redirected the phenotypic transformations of segmental structures in an opposite manner compared to the homeotic transformations observed in PcG mutants. The genes belonging to this class were termed "Trithorax group (TrxG)" and their products seemed to act as antirepressors that ensure a faithful expression activity of the master regulators (Kingston and Tamkun 2014).

While originally identified as regulators of homeotic genes in Drosophila, both genes of the PcG as well TrxG were subsequently found to be evolutionary highly conserved in plants and animals. Primarily by molecular homology, members of the two groups were identified in the genomes of a number of model organisms. In some way, this is not surprising as cellular memory is an inherent requirement for a multicellular organism. However, subsequent analyses found a much broader regulatory regimen for $\mathrm{PcG}$. They not only required to maintain developmental fates, but also used in a large number of other transcriptional control processes requiring maintenance of stable and heritable gene repression. The representatives of the TrxG are molecularly and functionally a rather diverse group and were found to be not only needed for many active transcriptional processes but also other nuclear processes requiring chromatin decompaction or activation. PcG and TrxG factors do not shape decisions for gene regulation, which is rather implemented by transcription factors (TFs) acting as master regulators. However, in many cases these TFs are switched off or take-up new tasks after their initiating signal. The further maintenance of the active or repressed state and the transmission of this information to the next cell generation is achieved by the combined action of PcG and TrxG. Hence, "cellular memory" could be understood as a mechanism for maintaining "transcriptional memory". Maintaining a transcriptional state in the absence of the initial trigger is a classical epigenetic task fulfilled by these factors. Indeed, subsequent studies using the corresponding technical advantages of the different model organisms under analysis, revealed a class of gene products with major activities in shaping and modulating chromatin structure and function in the nucleus. 


\subsection{Biochemical Characterization and Molecular Function of PcG/TrxG Proteins}

The molecular characterization of the genes and products of the PcG and TrxG provided deep insights into their specific role in the nucleus. General chromatin research had identified the important role of histones not only as packing material for the genome but as an additional carrier of information (see book Chap. 1 of Wutz). Histones depict an elaborate pattern of covalent modifications of their amino acids. Specific histone modifications were found to be correlated with defined transcriptional states, providing a platform for a gene-specific control and, therefore, producing an apparent code in addition to the genetic information encoded in the sequence of the DNA. An elaborate machinery consisting of chromatin factors writing, reading, and, if necessary, erasing the histone marks was revealed over the years (see book Chap. 1 of Wutz). Strikingly, among these so-called writers, readers, and erasers of epigenetic information, many were found to be encoded by PcG and TrxG genes.

Another interesting result was the discovery that PcG genes and to some degree also TrxG genes were genetically interacting. Subsequent molecular characterization revealed an interaction and cooperation of the proteins encoded by these groups in large and biochemically defined multimeric complexes. PcG proteins form two major types of complexes, Polycomb Repressive Complex 1 (PRC1) and 2 (PRC2) (Kuroda et al. 2020). Four PcG members constitute the core PRC1 complex in Drosophila: Polycomb (Pc), Polyhomeotic (Ph), Posterior sex combs (Psc), and Sex combs extra (Sce), also known as dRing1. PRC2 comprises four core proteins: Enhancer of zeste $(\mathrm{E}(\mathrm{z}))$, Suppressor of zeste $12(\mathrm{Su}(\mathrm{z}) 12)$, Extra sex combs (Esc), and Chromatin assembly factor 1, p55 subunit (Caf1-55), also known as p55 or Nurf55.

PRC1 recognizes and binds to the repressive H3K27me 3 mark via the chromodomain of Polycomb and blocks chromatin remodeling and transcription. PRC1 has also E3 ubiquitin ligase activity mediated by Sce/dRING1, which monoubiquitylates H2AK119 (in Drosophila the corresponding K118 is ubiquitylated). Through E(z)'s methyltransferase activity, PRC2 trimethylates lysine 27 on histone H3 (H3K27me3) at genes targeted for silencing, thus being responsible for this distinctive histone mark of $\mathrm{PcG}$ system-mediated repression. The $\mathrm{Su}(\mathrm{z}) 12$ and Esc subunits enhance this histone-modifying activity. The composition of the core complexes is conserved also in mammals (• Fig. 3.1). There, to deal with the higher regulatory complexity of the organism, a number of additional orthologs and factors cooperate with the core subunits to execute cell type- and tissue-specific functions. The concerted action of these protein complexes results in the stable and heritable transcriptional repression of PcG target genes.

The genetically defined group of TrxG proteins on the other hand was found to comprise a large and more divergent family of protein functions and complexes involved in the activation of transcription. They counteract PcG-mediated silencing by utilizing different mechanisms including the posttranslational modifications of histones, chromatin remodeling, recruitment of the transcriptional machinery, and 
Polycomb Repressive Complex 1 (PRC 1)
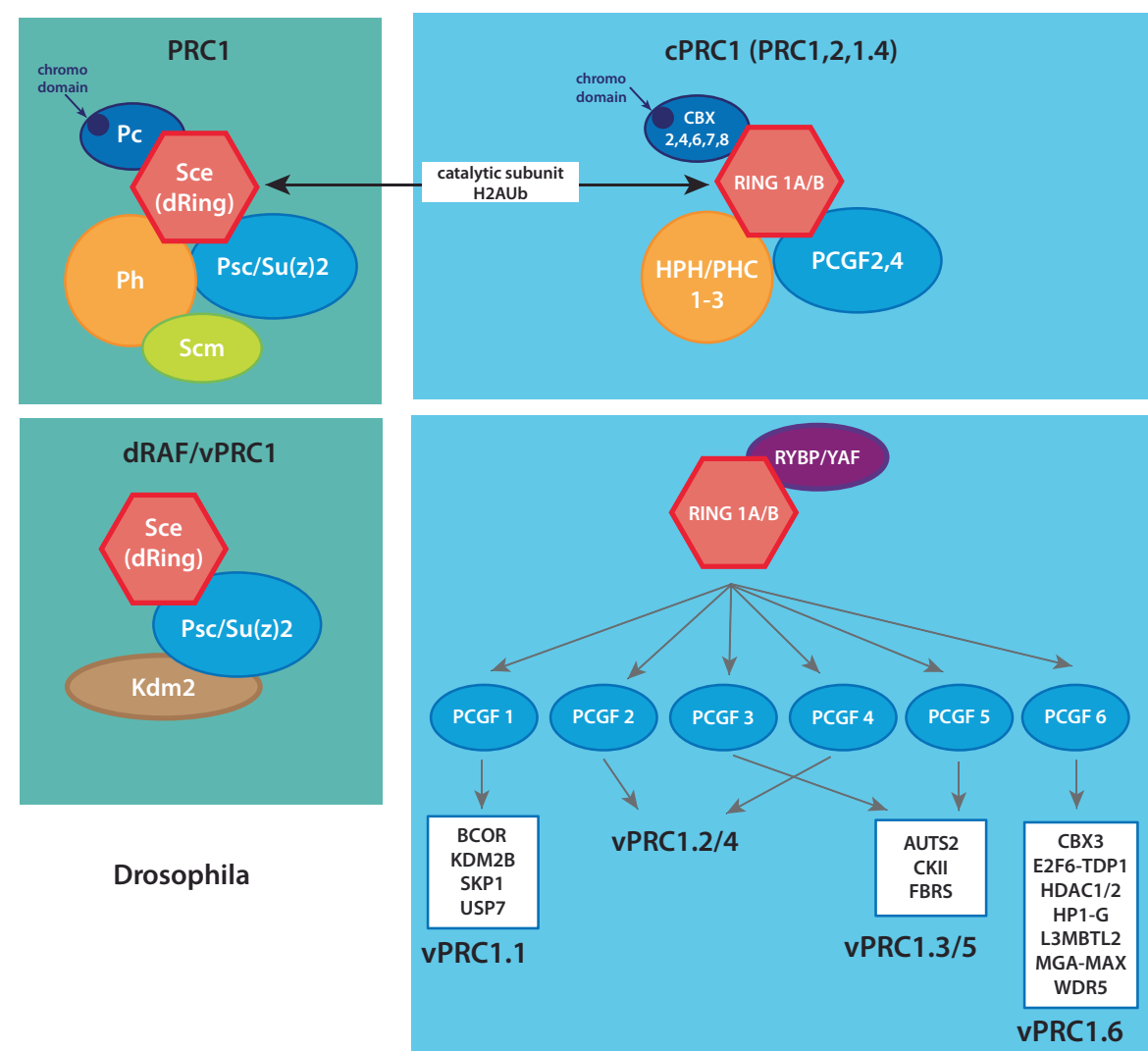

Mammals

Polycomb Repressive Complex 2 (PRC 2)

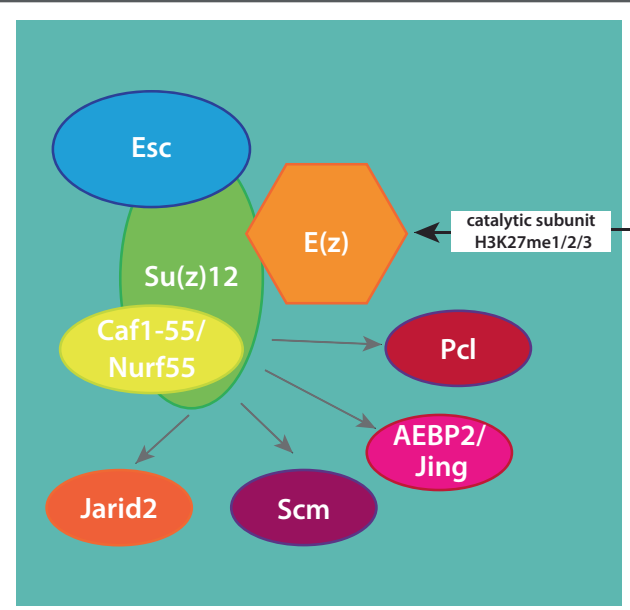

Drosophila

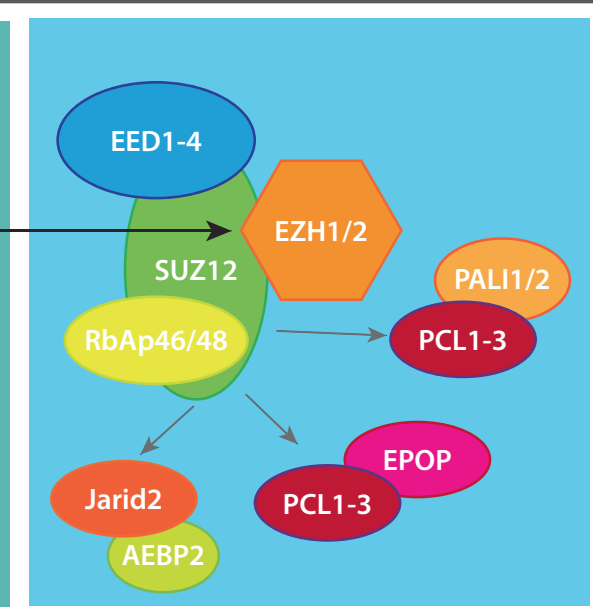

Mammals 
chromosome cohesion (Kingston and Tamkun 2014). The TrxG complexes TAC1, dCOMPASS, and dCOMPASS-like methylate $\mathrm{H} 3 \mathrm{~K} 4$, the modification that marks actively transcribed genes and active promoters. TrxG members also assemble into large SWI/SNF or BRG ATP-dependent chromatin remodeling complexes that can mobilize nucleosomes and promote chromatin accessibility (see book $>$ Chap. 2 by Paro).

Developmentally relevant TFs are often expressed in a tissue- and stage-specific manner. To a large degree, the activity of these TFs is controlled through their expression in the cell of action. Conversely, most of the core PcG and TrxG proteins are present in all cell types at all the time. Hence, the question is how these ubiquitously expressed factors, controlling transcriptional processes in a cell type specific manner, can maintain such an apparent specificity for their respective target genes.

\subsection{Targeting and Propagation of PcG/TrxG-Controlled Chromatin Domains}

In the Drosophila homeobox gene (HOX) clusters, the Antennapedia Complex (ANT-C) and BX-C, cis-regulatory elements attracting PcG proteins were identified. These so-called PcG Response Elements (PREs) can act in isolation by binding PcG proteins that silence adjacent reporter genes (Simon et al. 1993). Deletions of PREs result in ectopic mis-expression of the corresponding homeotic genes. Sequencespecific and globally expressed PcG factors like Pleiohomeotic (Pho) and GAGA factor (GAF) were found, among others, to provide the bridge between the DNA and PRC1 and PRC2, generating target specificity (Kuroda et al. 2020) (• Fig. 3.2). In the mammalian genome, targeting appears to use similar principles, albeit the exact nature and combination of TFs has not yet been determined. PcG complexes are enriched at unmethylated $\mathrm{CpG}$ islands. Various components, like KDM2, SUZ12 and JARID2, have affinity for GC-rich DNA sequences which may help to drive assembly of the complexes at nucleation sites. In addition, DNA methylation plays an important role in anchoring PRC2 to particular sites in mammals. Additionally, long non-coding RNAs have been suggested to anchor PcG complexes to target sites (see $>$ Chap. 4 of Wutz).

A major hallmark of PcG silencing is the H3K27me3 histone modification observed primarily at PREs and connected promoters but also extending largely over the body of the repressed gene. This "spreading" effect can be visualized using methods of chromatin immunoprecipitation (ChIP, see Methods Box 1 in book $>$ Chap. 1 of Wutz). The placement of the H3K27me3 mark over extended nucleosome regions can be explained by the combined and cooperative activities of individual

\footnotetext{
- Fig. 3.1 Polycomb Repressive Complex 1 and 2 in flies and mammals. PRC1 and PRC2 are biochemically identifiable multiprotein complexes. The core factors are color coded to show the respective counterparts in flies and mammals. The two complexes have a distinctive enzymatic activity; dRING or RING1A/B in PRC1 places a monoubiquitin at H2AK118 (Drosophila) and H2AK118 (mammals) and $\mathrm{E}(\mathrm{z})$ or EZH1/2 place a methyl moiety on $\mathrm{H} 3 \mathrm{~K} 27$. Some of the core proteins are found in variant complexes (vPRC1.1-6), especially in mammals. In combination with additional accessory factors these complexes fulfill specific tasks in a tissue-specific manner
} 


\section{Drosophila}
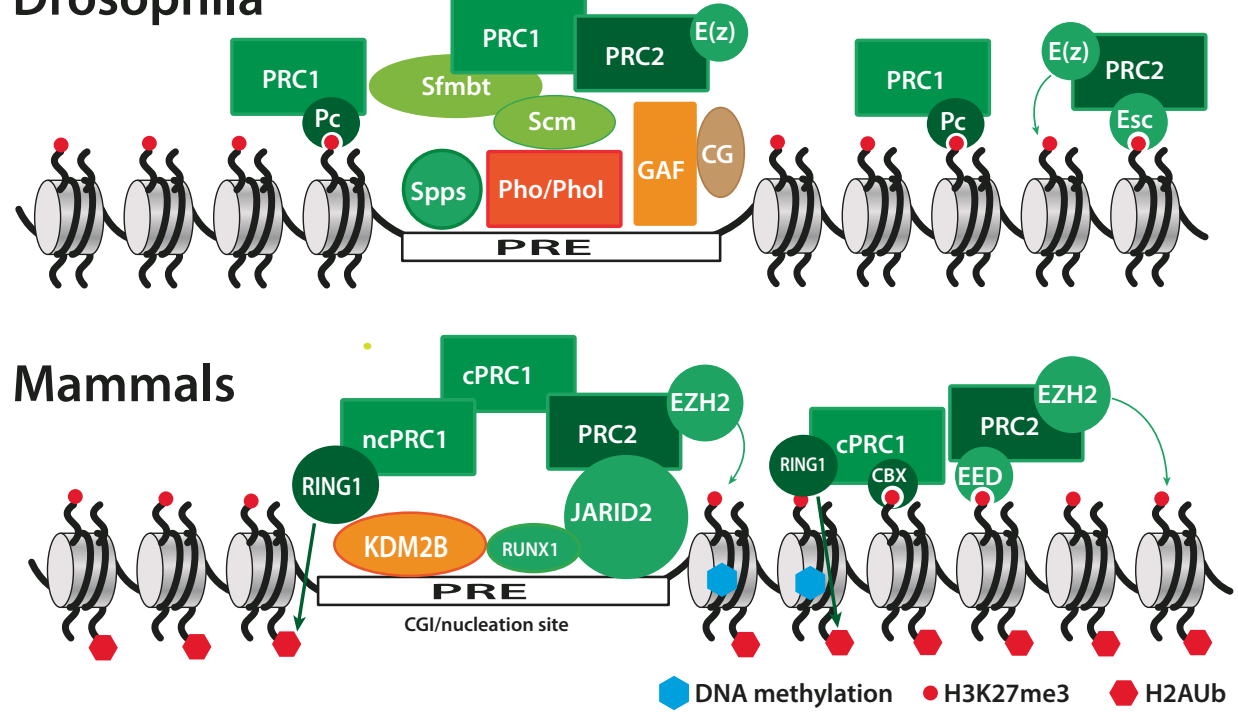

- Fig. 3.2 Anchoring of PcG complexes to silencing elements. (top) In Drosophila PcG repressive elements (PREs) are recognized and bound by a combination of sequence-specific TFs (Spps, Pho/Phol, GAF and CG). They interact with PRC2 complexes which produce the H3K27me3 signal, attracting PRC1. ESC/EED of PRC2 and PC/CBX of $\mathrm{cPRC} 1$ bind $\mathrm{H} 3 \mathrm{~K} 27 \mathrm{me} 3$ to drive self-propagation or spreading of the repressive mark. As a result, PREs can induce nucleosome compaction and inhibition of transcription by interaction with paused pol II. (bottom) In mammals the sequence requirement of PREs and corresponding TFs are less well understood. Unmethylated CpG islands attract PcG complexes through factors like KDM2, SUZ12 and JARID2 that have an affinity for CG-rich DNA. In contrast to Drosophila, where the role of H2Aub is not well understood, in mammals this mark facilitates recruitment of PRC2 via JARID2. PREs can also be used as platform for factors of the TrxG thereby acting as a switch for gene modulation

members of PRC1 and PRC2. The activity of the $\mathrm{H} 3 \mathrm{~K} 27$ histone methyltransferase $\mathrm{E}(\mathrm{z}) / \mathrm{Ezh}$ in PRC2 is stimulated by the other PRC2 component Esc/Eed that binds to H3K27me3, suggesting a feed-forward mechanism that could progress along the chromatin fiber. The Polycomb protein of PRC1 acts as a reader by binding the H3K27me3 mark through its chromodomain. In combination with other components of PRC1, like Polyhomeotic ( $\mathrm{Ph})$ containing multimerization modules (SAM domain), extended local clustering of chromatin sections are established. This leads to local nucleosomal condensation at regulatory sites and, in consequence, reduced DNA accessibility for TFs. Interestingly, such locally condensed regions can also interact with similar silenced regions in other parts of the genome, producing large, microscopically visible domains (Entrevan et al. 2016). An example for such longrange interactions can be found at the two Drosophila HOX clusters, normally separated by megabases on the linear chromosome, but colocalizing in the nucleus of cells where the Hox genes are repressed. The chemical property of certain components of the PcG induce the complexes to produce so-called phase-separated droplets, which have been termed PcG bodies. Such sub-nuclear compartments are not defined by molecular boundaries like membranes but, like oil and water, are separated by their disparate chemical properties. Recently, it was shown that the mammalian Polycomb component (CBX2) of canonical PRC1 can phase-separate in vitro and generate 
dynamic bodies in cells (Plys et al. 2019). The same mutations in CBX2 identified previously to impair nucleosome compaction and resulting in axial defects in mice were found to disrupt phase separation in vitro and the formation of bodies in cells. This brings together the ability to bind $\mathrm{H} 3 \mathrm{~K} 27 \mathrm{me} 3$, compact nucleosomes, and phase-separate into a single component of PRC1. These data support the hypothesis that phase separation and compaction by PRCl are generated by linked mechanisms. The authors therefore speculate that the high concentration of PRC1 within phaseseparated bodies could facilitate maintenance of a repressive chromatin state during development.

Multiplication of chromatin marks, multimerization of complexes, and clustering in the $3 \mathrm{D}$ space of the nucleus appear to be central requirements for generating stably and heritably repressed genomic domains. Indeed, the regulation of Hox genes, defining long-lasting cellular identities, nicely supports this restricted view of PcG function. However, most of the other PcG target genes identified change expression over time, the cell cycle or are even subjected to short-term environmental control. Here comes the family of TrxG factors into play. A surprising finding was that PREs do not only attract $\mathrm{PRC} 1 / 2$ proteins but also a number of TrxG proteins, suggesting they are switchable elements (PRE/TRE). While silencing by PcG components to gene-specific PREs represents the default state, a competitive off/on switch and finetuning is imposed by particular combinations of TrxG proteins and transcription factors that react to developmental or environmental cues (• Fig. 3.3).

An interesting case in this respect are so-called bivalent domains. Originally discovered in mammalian embryonic stem cells, they recently were also identified in the Drosophila embryonic genome (Akmammedov et al. 2019). Bivalent domains are characterized by the concomitant presence, over an extended genomic region, of the characteristic PcG histone mark H3K27me3 and the typical TrxG mark H3K4me3 (see also Methods Box 1 in book $>$ Chap. 1 of Wutz). In early embryonic stages, this dual marking at genes appears to signal a transcriptionally poised condition. Hence, a gene "waits" for the appropriate developmental signals to drive its expression into an active or repressed state, depending on the fate of the cell lineage. With ongoing developmental time and programming, the signals are resolved into either a complete H3K27me3 pattern (and/or in the case of mammals with additional DNA methylation signals) when the gene is repressed or a complete $\mathrm{H} 3 \mathrm{~K} 4 \mathrm{me} 3$ (and accompanying $\mathrm{H} 3 / \mathrm{H} 4$ acetylation) when the gene is active (Voigt et al. 2013). This site-specific antirepressor capability of members of the TrxG provides a means for how localized competition can switch the expression state from silenced to activated, also known as the "bivalent master switch" model (Kuroda et al. 2020). A central aspect is a shift of the balance by the addition of acetylation moieties, opening up nucleosomal condensation and allowing transcriptional activation. Indeed, PRC1 proteins have been found complexed with acetyltransferases and acetyl-binding bromo domain-containing proteins. Hence, the local balance between PcG and TrxG proteins defines the state of histone modifications and, consequently, the accessibility and processability of the chromatin template. However, based on theoretical considerations, it was suggested that poised chromatin is produced by a bistable state imposed by the PcG/TrxG competition rather than through a true bivalent marking of nucleosomes (Sneppen and Ringrose 2019). In their model, poised chromatin is bistable and not bivalent. They suggest that bivalent chromatin containing $\mathrm{H} 3 \mathrm{~K} 4 \mathrm{me} 3$ and $\mathrm{H} 3 \mathrm{~K} 27 \mathrm{me} 3$ is present as an unstable background population in all system states, and different subtypes co-occur 
a

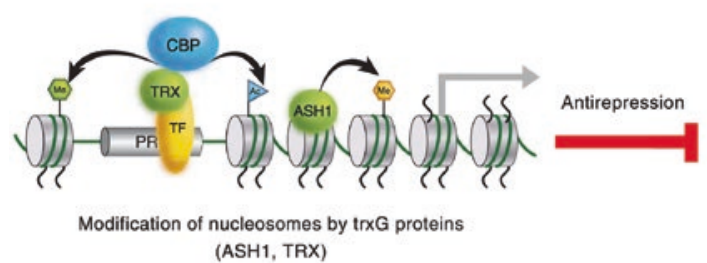

(ASH1, TRX)

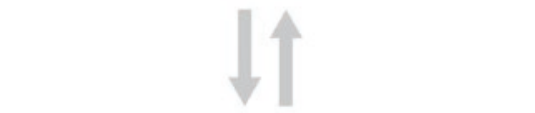

C

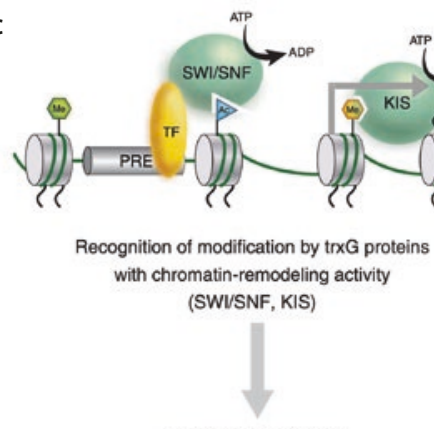

Active transcription

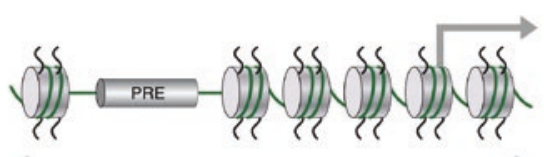

b

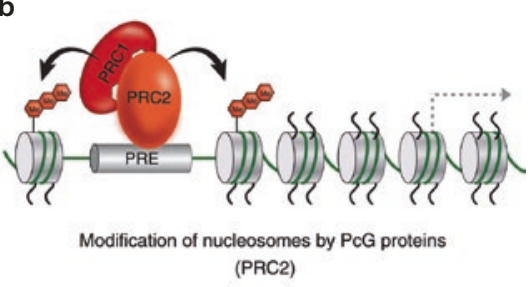

$\checkmark$ PRA

d

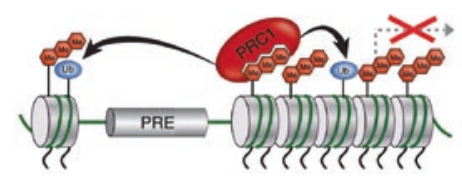

Recognition of modifications by PcG proteins (PRC1)

Heritable repression

$$
\text { H3K4me3 }
$$

- Fig. 3.3 Antagonistic functions between TrxG and PcG factors. Both TrxG and PcG families include proteins that covalently modify histones and those that non-covalently modify chromatin. Covalent modifications on histones can promote or block the binding or activity of TrxG complexes (e.g., SWI/SNF and KIS), PcG complexes (e.g., PRC1 and PRC2), or other factors involved in the maintenance of active or repressed states. Binding by these latter complexes has the potential to lead to further covalent modifications, thus leading to iterative cycles of covalent modification and recognition of the covalent marks. a TrxG factors are attracted to PREs at promoter regions and mark histones for activity. b In a different cell type the same gene is marked by negative histone modifications by PcG complexes bound at PREs. $\mathbf{c}$ Nucleosome remodeling complexes recognize the active histone marks and open nucleosomal packaging for access to the transcriptional machinery. $\mathbf{d}$ Modification of histones by $\mathrm{PcG}$ complexes result in compaction of nucleosomes and in gene repression. Depending on local constitution and concentration of the antagonistic factors, genes are either kept in an active or permanently repressed form. (from (Kingston and Tamkun (2014))

with active and silent chromatin. In contrast, bistability, in which the system switches frequently between stable active and silent states, occurs under a wide range of conditions at the transition between monostable active and silent system states. The question whether bistability and not bivalency is associated with poised chromatin has implications for the understanding of how the PcG/TrxG system maintains cellular 
memory. To resolve this important issue super resolution chromatin dissection analyses (single cell ChIP, single nucleosome analyses, etc.) will eventually be required.

A major interaction platform for both activities is the promoter of target genes. In particular, $\mathrm{PcG}$ proteins were found to act primarily at promoters with a paused Pol II. Pol II pausing is found at many protein-coding genes (see book $>$ Chap. 2 of Paro). Genome-wide ChIP analyses as well as Global Run-On sequencing (GROseq) studies indicated that the distribution of the PcG complexes PRC1 and PRC2 correlates significantly with genes exhibiting stalled polymerases, suggesting a common mechanistic basis. In murine ES cells, genes with bivalent domains and binding both PRC1 and PRC2 show substantially reduced levels of paused Poll II at the promoter region. This supports the view that nucleosomal compaction is part of the PcG-mediated silencing process. Conversely, many bivalent promoters with only PRC2 bound exhibit strong Pol II pausing at the 5'-end, suggesting that the PcG system overall controls important rate-limiting steps in assembly and transcriptional elongation at target genes. In Drosophila, many members of the TrxG are co-localized with PcG complexes at promoters, including those with paused promoters. Indeed, the occurrence of bivalent domains at particular mammalian genes and the cooccupancy of PRC1/2 components with TrxG proteins at many Drosophila target genes suggest a strong molecular interface between these opposing activities.

A still unresolved question in this respect is how this complex layering of regulatory levels, from local amplification of histone modifications, multimerization of protein complexes, to clustering in the $3 \mathrm{D}$ nuclear space, is reproduced after DNA replication and cell division. However, also in this case, the complexity might become reduced to the maintenance of a few relevant molecular marks, which subsequently act as entry sites to reconstruct the full epigenetic landscape. It has been suggested that $\operatorname{Tr} \mathrm{x}$ proteins (and/or local histone marks or activity) tag an active gene and still remain associated during S-phase where general chromatin remodeling is observed. Hence, they act as "bookmark" to keep the genes active which are necessary for the specific identity of the cell (Blobel et al. 2009). Conversely, in the ensuing G1 phase, all genes without a bookmark become silenced again by default and by the tagging through a PRE(s). This would ensure that only one part of the complex transcriptional memory machinery is required to be faithfully duplicated and actively transported through DNA replication and mitosis.

\subsection{Switching Memory and the Role of Non-coding RNAs}

The example of bivalent chromatin domains showed how PcG/TrxG competitive interactions lead to permanent opposing expression states necessary for developmental stability. However, many repressed genes need to become activated or active genes repressed at later developmental times or when required by physiological or environmental conditions. An example for the latter is the process of vernalization in plants (Costa and Dean 2019). Since plants are sessile, they need to adjust their growth, development, and physiology to the changing environment. To ensure that reproductive development only starts after the winter period and, therefore, during more favorable environmental conditions, plants developed mechanisms to "remember" that they experienced prolonged periods of cold. Central to this process is the epigenetic silencing of a floral repressor during cold periods. In Arabidopsis thaliana, the 

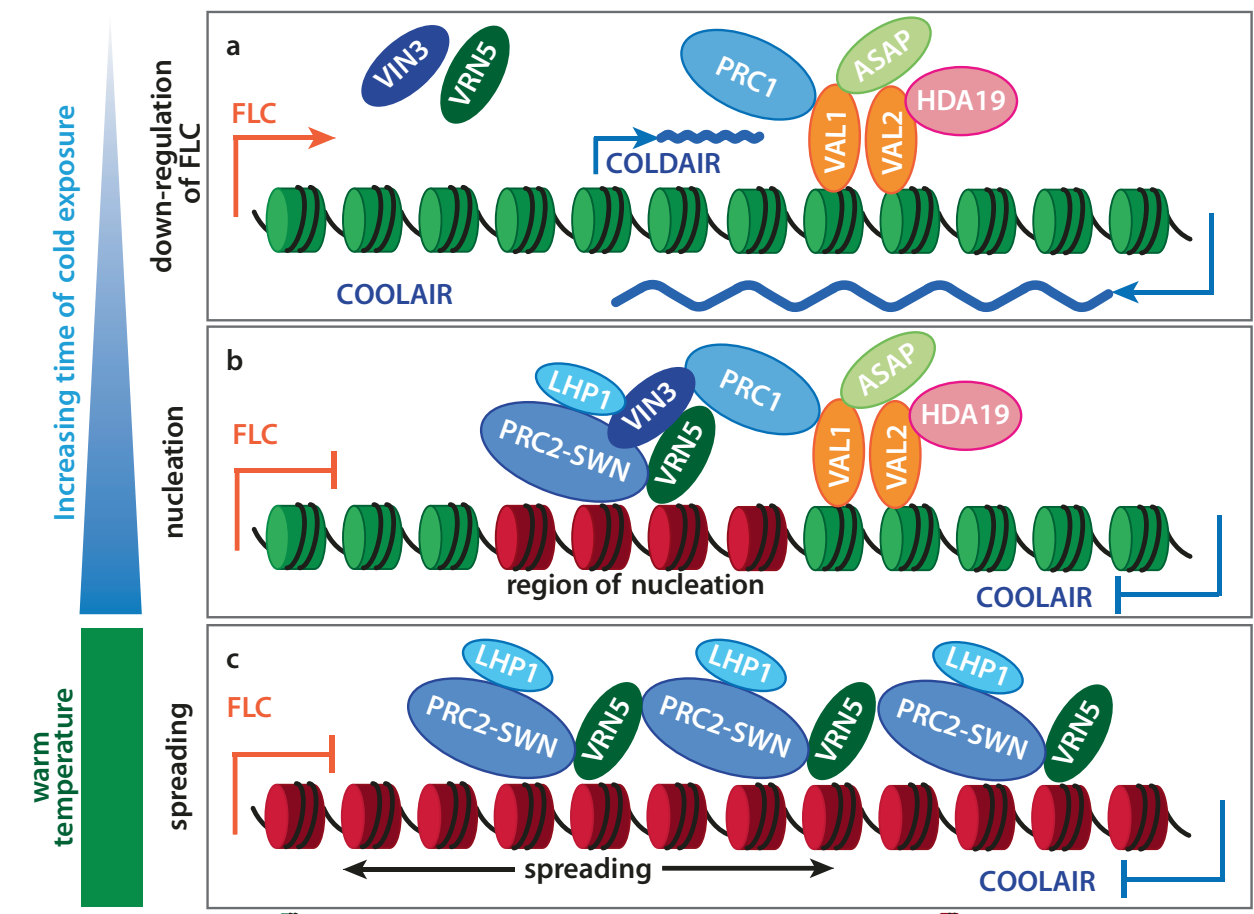

H3K4me2/3, H3K36me3 and acetylation

H3K27me3

- Fig. 3.4 Model for a lncRNA inducing an epigenetic switch at FLC. a Before cold exposure during winter time the FLC locus is expressed and the histones marked with $\mathrm{H} 3 \mathrm{~K} 4 \mathrm{me} 2 / 3$, H3K36me 3 and acetylation. At the start of cold exposure, the expression of the internal lncRNA Coldair attracts PcG complexes to the gene. Additionally, the anti-sense lncRNA Coolair becomes transiently expressed at high levels inducing the down-regulation of FLC transcription. Near the nucleation region VAL1/2 attract the histone deacetylase HDA19, the transcriptional regulator complex ASAP and PRC1, linking transcriptional repression to PcG silencing. b Nucleation start by ASAP associating with PRC2 accessory proteins VIN3 and VRN5 and with the PRC2-SWN complex. This generates a metastable silenced state of FLC starting accumulation of $\mathrm{H} 3 \mathrm{~K} 27 \mathrm{me} 3$ signals at the nucleation region. $\mathbf{c}$ After exposure to warm temperatures, this signal together with PRC2-CLF and LHP1 spreads over the entire locus establishing a long-term epigenetically silenced state. This condition is maintained across multiple cell division until reset in the next generation during embryogenesis

FLOWERING LOCUS C (FLC) transcription factor acts as a break to flowering. The expression of this repressor is gradually reduced during long periods of cold, so that, once plants detect inductive photoperiods and warm temperatures, the switch to flowering can be activated. The repression of FLC is stable and maintained for many months after cold exposure until embryogenesis in the seed producing the next generation, leading to reactivation of $F L C$ (• Fig. 3.4).

The discovery of a pair of long non-coding RNAs (lncRNAs) transcribed at the $F L C$ locus revealed a novel role for such transcripts in an epigenetic switch mechanism. Cold induces the local accumulation of both lncRNAs: COLDAIR, which is transcribed inside an intron of $F L C$, and COOLAIR, which is transcribed antisense to the FLC mRNA. COLDAIR recruits plant PRC2 containing the E(z) homologs CURLY LEAF (CLF) and SWINGER (SWN). This results in enrichment of H3K27me at the $5^{\prime}$ and $3^{\prime}$ ends of FLC. The antisense COOLAIR transcript spans 
the FLC gene and promotes removal of the activating H3K4me and H3K36me marks. The lncRNAs trigger the repression of $F L C$, which is subsequently stabilized by $\mathrm{PcG}$ silencing. Like at PREs, PRC2 complexes are concentrated at a single site inside the FLC gene which acts as a nucleation site for $\mathrm{H} 3 \mathrm{~K} 27 \mathrm{me} 3$ spreading. Eventually, the entire FLC chromatin is covered by PRC2 and the accompanying histone methylation mark. Interestingly, the degree of methylation at the nucleation site as well as the extent of the silenced domain correlate with the length of the cold period, providing a rheostat for the vernalization process.

The role of lncRNAs play an ever-increasing importance in epigenetic processes and, in particular, interactions with the PcG silencing complexes have been extensively documented. One such example is XIST involved in mammalian X chromosome inactivation (see book $>$ Chap. 4 of Wutz). Other examples include the HOTAIR lncRNA involved in silencing Hox genes in the mammalian genome (Achour and Aguilo 2018). HOTAIR acts in trans to repress expression. The lncRNA is transcribed from the $H O X C$ locus on chromosome 12 but recruits PRC2 and the LSD1-containing coREST/REST complex to HOXD on chromosome 2 . Recruitment leads to deposition of $\mathrm{H} 3 \mathrm{~K} 27$ me by $\mathrm{PRC} 2$ and removal of activating $\mathrm{H} 3 \mathrm{~K} 4 \mathrm{me}$ marks by LSD1. In these cases, the RNAs appear to act as scaffolds either in cis or in trans for the silencing activity. However, regulatory modules have been described where the transcription of a lncRNA through a PRE leads to the displacement of the silencing complexes and in consequence to the activation of a target gene (Ringrose 2017). In animals, reports where the sequence of a lncRNA, by its complementarity to DNA, is used to target epigenetic complexes to specific genomic sites are not well validated and still controversial. This is in contrast to small RNAs in plants that can clearly recruit chromatin and DNA modifying complexes to target sites in a sequencespecific manner (see book Chap. 6 of Grossniklaus).

In summary, there are several cases where the act of transcribing a lncRNA (or any other transcript) can displace a PcG silencing complex. In parallel, transcripts exist which could locally attract PcG complexes in cis and induce the silencing of a gene. This offers the potential of a switch mechanism, based on the activity of RNAs. Indeed, the role of lncRNAs acting in cis in switching PcG/TrxG transcriptional states has been modelled based on published criteria (Ringrose 2017). Depending on the kinetic parameters, lncRNAs can act to recruit PRC2 to target chromatin or can be induced to evict PRC2 from silenced chromatin (see $\bullet$ Fig. 3.5).

\subsection{Losing Memory}

A natural form of cellular memory loss is required during the transmission of genetic information by the germ cells for fertilization and generation of progeny. Germ cells need to strap any epigenetically encoded information covered by tissue-specific chromatin domains or epigenetically imprinted marks caused by environmental cues (Reik and Surani 2015). Indeed, development of germ cells encompasses several bottlenecks where chromatin information is apparently erased to ensure a faithful re-start of transcriptional programming at fertilization. These range from the replacement of histones with protamines, followed by a strong compaction of the genome in sperm cells, to the complete genome-wide DNA demethylation observed in early mammalian preimplantation embryos (see book Chap. 6 of Grossniklaus). 
a

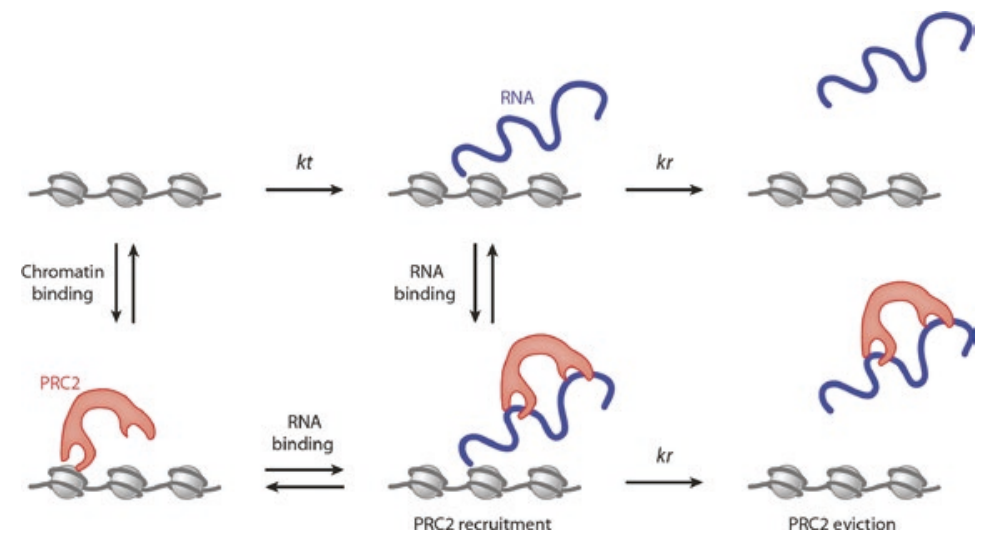

b Slow RNA release: PRC2 recruitment and silencing

C Fast RNA release: PRC2 eviction and activation
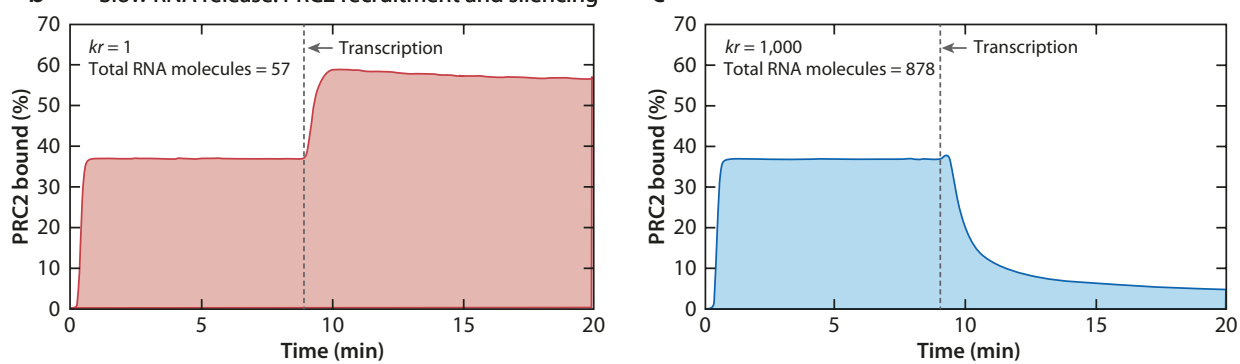

- Fig. 3.5 Simple kinetic model for interaction of PRC2 with RNA and chromatin. a The model is based on ordinary differential equations and contains three components: chromatin (gray), PRC2 (red), and RNA (dark blue). (Left) Chromatin binding: PRC2 can bind directly to chromatin. The dissociation constant $(\mathrm{Kd})$ for this interaction is $86 \mathrm{nM}$, measured for PRC2 binding to H3K27me 3 modified histone tails. (Middle) RNA binding: PRC2 can also bind to RNA. The Kd used for this interaction in the simulations shown is $30 \mathrm{nM}$. RNA is produced with rate kt, released from chromatin with rate $\mathrm{kr}$, and degraded with rate kd (not shown). (Right) RNA is released from chromatin after transcription, either unbound (top) or bound (bottom) by PRC2. b, c The system was simulated over a time course, and RNA transcription was initiated after $8 \mathrm{~min}$. The input quantities for these simulations were set to reflect average molecule numbers in a $0.1 \mu^{3}$ volume in a Drosophila embryo nucleus at cycle 12 , based on total nuclear concentrations (approximately the confocal volume used for fluorescence correlation spectroscopy experiments. The quantities were PRC2 $=5$ molecules; H3K27me $3=52$ molecules, assuming $6 \%$ of tails modified; $\mathrm{kt}=0.2 \mathrm{~s}^{-1}$; and $\mathrm{kd}=0.01 \mathrm{~s}^{-1}$. $\mathrm{kr}$ was varied as shown in panels $\mathrm{b}$ and $\mathrm{c}$. RNA molecules were assumed to be localized in the volume of the simulation. (From Ringrose (2017))

Interestingly, exceptions have been observed in both animal and plant reproduction where not all epigenetic information is erased, influencing the normal growth and physiology of the progeny. A first case was described in Drosophila with a PRE controlling a white reporter gene (responsible for the red eye color in flies) (Ciabrelli et al. 2017; Cavalli and Paro 1998). Normally, the PRE would silence the reporter resulting in white-eyed flies, given that the endogenous white locus was mutant. Through a single transcriptional switch, the reporter gene could become activated and stay on through the entire development of the fly. Surprisingly, the activated form was not erased in the germ line but transmitted to the progeny, which also displayed red eye color. The effect could be maintained over several generations, demonstrating that, under specific conditions, a single transcriptional signal, changing the modularity of 
PREs, can propagate epigenetically encoded information over a number of generations. Instances of transgenerational inheritance have now been observed also in mammals (Boskovic and Rando 2018). One of the most influential examples is the transgenerational inheritance of the expression state of the agouti gene, responsible for coat color, in mice (see also Book $>$ Chap. 9 of Santoro). Specific diets of parents can change the DNA methylation pattern at the locus which, in the germline, is not always erased and thus propagated to the offspring. The transferred epigenetic mark controls the expression of the agouti gene and, hence, the coat color in the next generations. While transmission through meiosis, gametogenesis, and early embryogenesis based on DNA methylation marks are well documented, in many other cases the underlying epigenetic signal that is transmitted and causes an effect in the progeny is not always clear (Grossniklaus et al. 2013). Conversely, in plants, transgenerational epigenetic regulation in response to environmental stress is well described both at the physiological and molecular level.

Cancer is largely considered a genetic disease. With accumulating mutations in oncogenes or tumor suppressor genes, the cells lose their contacts with the neighbors, start to over-proliferate, change metabolic behavior, and eventually migrate to create the deadly metastases. Cancer is also a disease of transcriptional mis-regulation and, given the role of chromatin in this process, it is not surprising to realize that many mutations in chromatin-controlling factors have been observed in human cancers. Mutations ensure the long-term phenotypic change of cancer cells. However, epigenetic marks like DNA methylation or histone modifications can also be long-lasting, maintaining gene expression states over many cell generations. In consequence, deregulated expression in cancer cells need not only have a mutational basis but is often driven by epigenetic mis-regulation. In $>$ Chap. 9 cancer epigenetics is described in great detail. Here, two examples for mutations in components of the cellular memory system will be outlined, illustrating how the loss of cellular identity during ontogeny can lead to the development of a tumorigenic state.

The human TrxG gene Mixed Lineage Leukemia (MLL1) was initially identified as a chromosomal translocation found in blood cells and causing acute lymphoblastic or myeloid leukemia. MLL1 codes, as its Drosophila ortholog trithorax (trx), for a histone 3 lysine 4 methyltransferase (KMT), catalyzing the addition of the positive histone mark $\mathrm{H} 3 \mathrm{~K} 4 \mathrm{me} 3$. The translocation brakes inside the gene, often fusing the $\mathrm{N}$-terminal part of the MLL1 protein with other transcriptional control factors. The C-terminal part containing the KMT activity (SET domain) is removed, depriving MLL1 from its normal activating function. Depending on the fusion partner, MLL1 can repress or activate target genes in an aberrant manner and thereby induce cancertypical expression profiles. Since the KMT activity normally repels the DNA methylation machinery, the MLL1 fusions, where this part is often absent, are often found at hypermethylated promoters. This results in the down-regulation of many target genes. Conversely, the N-terminal part of MLL1 contains a DNA-binding affinity. The recruitment of co-activators through the fused partner protein can result in the hyperactivation of target genes. The result is a highly deregulated gene expression profile found in leukemic cells. By studying the molecular partnerships of MLL1 fused, for example, to the AF9 activator, specific small molecule inhibitors could be identified. In one case, MLL1 interacts with the histone demethylase LSD1. When blocked by a small inhibitor (LSD1i) the aberrant activating function of the fusion MLL1 is reduced. Similarly, preventing another interaction partner, BRD4, to bind 
via its bromo domain to acetylated histones by blocking the reader function (BETi) also reduces the transcriptional hyperactivity (see also book $>$ Chap. 8 of Santoro). Hence, the detailed knowledge of the molecular processes encompassing TrxGdriven anti-repressor activity allowed the development of highly specific inhibitors, which are currently in clinical trials as anti-cancer drugs.

Mutations in PcG genes are often associated with a variety of human cancers of a large range of cell types and tissues. Similarly, down-regulation of members of the PcG protein complex in Drosophila can lead to neoplastic growth in imaginal discs (Beira et al. 2018). The role of the PRCl component polyhomeotic (ph) has been studied in substantial detail, revealing how loss of cellular identity can drive cells to an over-proliferative behavior. Interestingly, no genetic instability is observed in these tumor cells. Epigenetic dysregulation reprograms the cells to an embryonic state and blocks them from entering differentiation. However, by enforcing development through the overexpression of a differentiation factor, tumorigenic competence is restrained (Torres et al. 2018). Not only in fly cancers but also in many human cancers, cells with overproliferative traits often depict a de-differentiated state. This reflects the characteristics and need of these cells to divide out of control, independent of the neighboring environment and developmental cues. Interestingly, novel approaches, termed "differentiation therapy", attempt to force cancer cells into the differentiation path again, eventually removing them from the pool of overproliferating cells. Indeed, a large number of new cancer drugs that are currently in clinical trials target epigenetic factors, attempting to reverse aberrant transcriptional profiles (see also book $>$ Chap. 8 of Santoro) (Audia and Campbell 2016).

In summary, the chromatin factors encoded by the PcG and TrxG are required for maintaining developmental and other transcriptional states. While decisions to acti-

\section{Take-Home Message}

- The PcG/TrxG system, including the chromatin modifying and remodeling activities, is evolutionary highly conserved and is involved in many basic cellular processes like maintenance of developmentally controlled gene expression states (cellular memory), mammalian $\mathrm{X}$ chromosome inactivation, and vernalization in plants.

- PcG multiprotein complexes are involved in long-term silencing of genes. $\mathrm{H} 3 \mathrm{~K} 27 \mathrm{me} 3$ is a hallmark of $\mathrm{PcG}$ repression, correlated with condensed nucleosomal structures. PcG repressed domains cluster in the $3 \mathrm{D}$ space of the nucleus to produce stable silencing.

- TrxG complexes counteract PcG repression and are required for maintaining gene activity stable and heritable. H3K4me3 is the hallmark of the TrxG, which encodes writers and readers of histone modifications but also components of the nucleosome remodeling complexes involved in opening chromatin structures.

- Long non-coding RNAs play an important role in the silencing mechanism of the PcG. It has been suggested that lncRNAs interact with PcG protein complexes to add structural features, evict PRCs from PREs, or target the complexes to specific genomic sites.

- Deregulation of components of the PcG/TrxG system often results in cancer development. Epigenetic cancers are not dependent on the accumulation of genetic aberrations. Many new drugs against chromatin regulators are currently being tested in the clinic. 
vate or silence a gene are made by master regulators, the resulting expression state can be maintained and faithfully inherited from one cell generation to the next by the epigenetic landscape imposed by either PcG- or TrxG-driven marks. The competitive and bistable nature of PcG and TrxG allows, however, the reversion of expression states if required, producing a highly versatile and adaptive regulatory network.

\section{References}

Achour C, Aguilo F (2018) Long non-coding RNA and Polycomb: an intricate partnership in cancer biology. Front Biosci 23:2106-2132

Akmammedov A, Geigges M, Paro R (2019) Bivalency in Drosophila embryos is associated with strong inducibility of Polycomb target genes. Fly 13(1-4):42-50. https://doi.org/10.1080/19336934.2019.1 619438

Audia JE, Campbell RM (2016) Histone modifications and cancer. Cold Spring Harb Perspect Biol 8(4):a019521. https://doi.org/10.1101/cshperspect.a019521

Beira JV, Torres J, Paro R (2018) Signalling crosstalk during early tumorigenesis in the absence of Polycomb silencing. PLoS Genet 14(1):e1007187. https://doi.org/10.1371/journal.pgen.1007187

Blobel GA, Kadauke S, Wang E, Lau AW, Zuber J, Chou MM, Vakoc CR (2009) A reconfigured pattern of MLL occupancy within mitotic chromatin promotes rapid transcriptional reactivation following mitotic exit. Mol Cell 36(6):970-983. https://doi.org/10.1016/j.molcel.2009.12.001. S1097-2765(09)00904-6 [pii]

Boskovic A, Rando OJ (2018) Transgenerational epigenetic inheritance. Annu Rev Genet 52:21-41. https://doi.org/10.1146/annurev-genet-120417-031404

Cavalli G, Paro R (1998) The Drosophila Fab-7 chromosomal element conveys epigenetic inheritance during mitosis and meiosis. Cell 93(4):505-518

Ciabrelli F, Comoglio F, Fellous S, Bonev B, Ninova M, Szabo Q, Xuéreb A, Klopp C, Aravin A, Paro R, Bantignies F, Cavalli G (2017) Stable Polycomb-dependent transgenerational inheritance of chromatin states in Drosophila. Nat Genet 81:83. https://doi.org/10.1038/ng.3848

Costa S, Dean C (2019) Storing memories: the distinct phases of Polycomb-mediated silencing of Arabidopsis FLC. Biochem Soc Trans 47(4):1187-1196. https://doi.org/10.1042/BST20190255

Entrevan M, Schuettengruber B, Cavalli G (2016) Regulation of genome architecture and function by polycomb proteins. Trends Cell Biol 26(7):511-525. https://doi.org/10.1016/j.tcb.2016.04.009

Grossniklaus U, Paro R (2014) Transcriptional silencing by polycomb-group proteins. Cold Spring Harb Perspect Biol 6(11):a019331. https://doi.org/10.1101/cshperspect.a019331

Grossniklaus U, Kelly B, Ferguson-Smith AC, Pembrey M, Lindquist S (2013) Transgenerational epigenetic inheritance: how important is it? Nat Rev Genet 14(3):228-235. https://doi.org/10.1038/ $\operatorname{nrg} 3435$

Hadorn E (1968) Transdetermination in cells. Sci Am 219(5):110-114. passim

Kingston RE, Tamkun JW (2014) Transcriptional regulation by trithorax-group proteins. Cold Spring Harb Perspect Biol 6(10):a019349. https://doi.org/10.1101/cshperspect.a019349

Kuroda MI, Kang H, De S, Kassis JA (2020) Dynamic competition of polycomb and trithorax in transcriptional programming. Annu Rev Biochem 89(1):235-253. https://doi.org/10.1146/annurev-biochem-120219-103641

Lewis EB (1978) A gene complex controlling segmentation in Drosophila. Nature 276:565-570

Plys AJ, Davis CP, Kim J, Rizki G, Keenen MM, Marr SK, Kingston RE (2019) Phase separation of Polycomb-repressive complex 1 is governed by a charged disordered region of CBX2. Genes Dev 33(13-14):799-813. https://www.genesdev.org/cgi/doi/10.1101/gad.326488.119

Reik W, Surani MA (2015) Germline and pluripotent stem cells. Cold Spring Harb Perspect Biol 7(11). https://doi.org/10.1101/cshperspect.a019422

Ringrose L (2017) Noncoding RNAs in polycomb and trithorax regulation: a quantitative perspective. Annu Rev Genet 51(1):385-411. https://doi.org/10.1146/annurev-genet-120116-023402

Simon J, Chiang A, Bender W, Shimell MJ, O'Connor M (1993) Elements of the Drosophila bithorax complex that mediate repression by Polycomb group products. Dev Biol 158(1):131-144 
Sneppen K, Ringrose L (2019) Theoretical analysis of Polycomb-Trithorax systems predicts that poised chromatin is bistable and not bivalent. Nat Commun 10(1):2133. https://doi.org/10.1038/s41467019-10130-2

Struhl G, Akam M (1985) Altered distributions of Ultrabithorax transcripts in extra sex combs mutant embryos of Drosophila. EMBO J 4(12):3259-3264

Torres J, Monti R, Moore AL, Seimiya M, Jiang Y, Beerenwinkel N, Beisel C, Beira JV, Paro R (2018) A switch in transcription and cell fate governs the onset of an epigenetically-deregulated tumor in Drosophila. elife 7:777. https://doi.org/10.7554/eLife.32697

Voigt P, Tee W-W, Reinberg D (2013) A double take on bivalent promoters. Genes Dev 27(12):13181338. https://doi.org/10.1101/gad.219626.113

Wolpert L, Tickle C, Martinez AC (2015) Principles of development. Oxford University Press, Oxford

Open Access This chapter is licensed under the terms of the Creative Commons Attribution 4.0 International License (http://creativecommons.org/licenses/by/4.0/), which permits use, sharing, adaptation, distribution and reproduction in any medium or format, as long as you give appropriate credit to the original author(s) and the source, provide a link to the Creative Commons license and indicate if changes were made.

The images or other third party material in this chapter are included in the chapter's Creative Commons license, unless indicated otherwise in a credit line to the material. If material is not included in the chapter's Creative Commons license and your intended use is not permitted by statutory regulation or exceeds the permitted use, you will need to obtain permission directly from the copyright holder. 